(see C. Bycroft et al. Nature 562, 203-209; 2018). In a second study, researchers report brain imaging of 10,000 individuals, which reveals genetic influences on brain structure and function, and shows correlations with neurodegenerative, psychiatric and personality traits (see L. T. Elliott et al. Nature 562, 210-216;2018). Such findings are invaluable, but the usefulness of the UK Biobank project goes beyond immediate clinical relevance. It offers lessons for researchers establishing population-cohort and genomic-medicine projects elsewhere.

The UK Biobank benefits greatly from the infrastructure and centralization of the United Kingdom's National Health Service (NHS). In addition to recruitment through NHS centres, the project follows participants by accessing their health records and national registries, including those for deaths and cancer diagnoses.

Notably, the UK Biobank is the first project to demonstrate the successful collection and sharing of linked genetic, physical and clinical information on a population scale. All involved should thank the 500,000 volunteers across the United Kingdom who responded to their invitations and agreed to contribute their time, samples and health information. Buoyed by this success, UK Health Secretary Matt Hancock last week confirmed a significant expansion of genomic medicine in the NHS, which will grow the 100,000 Genomes Project to sequence the genomes of 1 million people through the NHS and the UK Biobank. This is part of an even more ambitious project to sequence up to 5 million genomes over the next 5 years, including those of seriously ill children and people with rare types of cancer.

Such scale is important, but so is diversity. The UK Biobank is filled with people who lived near an assessment centre and agreed to participate. Aiming for a more diverse population is an additional challenge, but a worthy one. The All of Us cohort study in the United States is making efforts to do this with targeted recruitment.

In many population-cohort studies, the data are not made accessible to other researchers until the initial findings are published, and even then only a few make their full data sets available. The UK Biobank, funded primarily by the Medical Research Council and the Wellcome Trust, and run as a charity, has taken an important stand. It has generously made its full data sets, as well as all results from studies conducted by researchers using these data, available from the outset.

The value of such an open approach is clear.

"That is the
future of
medicine:
wisdom from
crowds."
Since the UK Biobank opened general access to its database in March 2012, there have been at least 8,294 approved registrations, and 796 formally registered projects are under way. The results of these studies have been communicated in more than 500 publications in peer-reviewed journals and in over 100 preprints on a dedicated bioRxiv channel.

In particular, this access has allowed researchers to quickly search for genetic associations for a large and diverse collection of clinically relevant traits. A News Feature on page 181 explores what we have learnt from these larger-scale studies about genetic risk of disease, particularly the development of risk scores involving multiple genes, which could help to guide preventive measures for some common ailments such as coronary artery disease. Although controversial, such tests are already being developed commercially.

Many of these studies have aggregated UK Biobank data with other data sets to enable studies on a much larger scale, some reaching more than 1 million individuals. That is the future of medicine: wisdom from crowds.

\section{Noble effort}

\section{The bodies that govern the Nobel prizes must do more to achieve equality.}

$\mathrm{N}$ ewly minted Nobel laureates Donna Strickland and Frances Arnold are outstanding scientists. They are also women. Advocates of equality in science understandably feel torn between celebrating these women's achievements and shouting that their triumph does not mean the problem of equality in science is solved. The day when attributes such as the gender, sexuality or ethnicity of a Nobel prizewinner is not relevant will be a great one, but it is not today. The bodies that govern the Nobel prizes must to do more to achieve that.

Gender is an area in which the Nobel skew is particularly obvious. An abysmally small number of women have been awarded one of the science Nobel prizes - this year's awards bring the tally to 19 women out of 607 laureates (including people who have won two science Nobels) - still just $3 \%$, or $9 \%$ over the past decade. This is important because, for good or ill, the Nobel prizes matter. Laureates become science superstars and role models whose voices are amplified overnight. The prizes signal to the public who is the best of the best. Many of them recognize work from a time when the representation of women and people of colour was even lower than it is today. But, crucially, the awards are part of a system in which the balance remains tipped in the favour of Western white men, not just a product of that system.

Commendably, the bodies in Stockholm that award the prizes - the Royal Swedish Academy of Sciences for chemistry and physics, and the Nobel Assembly at the Karolinska Institute for physiology or medicine - have recognized that there are more people from under-represented groups who deserve the prize than receive it. And changes that the bodies introduced this year, which will take effect in 2019, could help. (These include flagging to nominators that they can select multiple candidates, and that they should consider diversity in gender, geography and topic - both of which encourage nominators to look beyond their immediate biases.)

But if the committees receive too few women candidates, why not highlight this by publishing aggregate demographic data on nominations? Right now, we know nothing about whether two female science winners in 2018 is a blip or representative of shifting attitudes, nor at what stage of the process the problem really lies - in nominations or selections. Having data on any problem is the first stage in tackling it. Transparency is a growing movement in science - and rightly so. Similar efforts can be made for scientific prizes.

Instead, the Nobel committees hide such information behind statutes that say nominations must remain confidential for 50 years. A spokesperson for the Royal Swedish Academy of Sciences told Nature that this is to prevent attempts to interfere with the nomination process and to allow researchers to give their honest opinion on colleagues' work. But it is hard to see how revealing aggregate data threatens that. Moreover, Alfred Nobel's will, on which these rules are based, does not call for confidentiality - merely stipulating that "the prize be awarded to the worthiest person". In this case, changing the statutes or their interpretation to allow for greater transparency will only help to achieve that.

The committees should also look at their own diversity. And they should state robustly why deserving women rarely win: because biases that are baked into the scientific system subtly (and sometimes not so subtly) hinder their route to the top as well as their eventual recognition. Evidence squarely shows this (see page 165). The situation is compounded for scientists who are from sexual and gender minorities, who are people of colour, who are disabled or not from a Western country. The world is still waiting for the first black winner of a science Nobel.

The march of history is towards equality, and many more like Strickland and Arnold are no doubt waiting in the Nobel wings. These women's wins are sources of hope for those who come after them. Strickland says that she feels she has been treated with equality in her career, a point that should be celebrated. But when women winning is not unusual enough to provoke comment - that will be the day for true celebration. 